\title{
Lipochoristoma of the Internal Auditory Canal
}

\author{
George Scangas ${ }^{1}$ Aaron Remenschneider ${ }^{1}$ Felipe Santos $^{1}$ \\ ${ }^{1}$ Department of Otolaryngology, Massachusetts Eye and Ear Infirmary, \\ Harvard Medical School, Boston, Massachusetts, United States \\ J Neurol Surg Rep 2015;76:e52-e54.

\begin{abstract}
Address for correspondence George Scangas, MD, Department of Otolaryngology, Massachusetts Eye and Ear Infirmary, Harvard Medical School, 243 Charles Street, Boston, MA 02114, United States (e-mail: george_scangas@meei.harvard.edu).
\end{abstract}

\begin{abstract}
Objective Lipochoristomas comprise $0.1 \%$ of all cerebellopontine angle tumors and may be misdiagnosed as vestibular schwannomas. We describe a lipochoristoma of the internal auditory canal (IAC) and present the salient features of the evaluation, diagnosis, and management.

Study Design and Methods Retrospective case review.

Results (Case Report) A 51-year-old woman presented with left-sided severe hearing loss, tinnitus, mild episodic vertigo, and facial paresthesias progressive over 1 year. Magnetic resonance imaging (MRI) demonstrated a small $(5 \times 4 \mathrm{~mm})$ T1 hyperintense lobulated lesion in the distal internal auditory canal. With fat-suppressed images, there was no enhancement of the lesion. A diagnosis of IAC lipochoristoma was made. Conservative management was recommended, and on 17-month follow-up there was no interval growth. The patient remains symptomatically stable with improved equilibrium but persistent left-sided hearing loss.

\section{Keywords}

- lipochoristoma

- internal auditory canal

- cerebellopontine angle

- acoustic neuroma

Conclusions Differential diagnosis of an enhancing lesion in the IAC includes acoustic neuroma, meningioma, epidermoid and arachnoid cysts, lipochoristoma, and metastatic tumors. Fat-suppressed MRI sequences aid in a definitive diagnosis of lipochoristomas. Because lipochoristomas may have a tendency for more indolent growth and intimate involvement of the auditory nerve, conservative management with interval imaging is recommended. Surgical treatment is reserved for growing lesions or those with disabling vestibular symptoms.
\end{abstract}

\section{Introduction}

Neoplasms arising within the internal acoustic canal (IAC) and cerebellopontine angle (CPA) are most commonly of neuroepithelial origin. Vestibular schwannoma represents the most common lesion in the IAC (80-90\%) with meningiomas comprising nearly all the rest (10\%) of IAC tumors. ${ }^{1-3}$ Rare tumors include epidermoids, lipochoristomas, and metastatic tumors. Each of these lesions has typical imaging characteristics that frequently allow a diagnosis to be made with magnetic resonance imaging (MRI) scan alone.

Lipochoristomas (lipomatous choristomas) comprise $0.1 \%$ of all CPA tumors. These rare tumors of the CPA and IAC are slow growing and often discovered incidentally. Classically, these tumors were thought to arise from cells of the meninx primitiva, the mesenchymal derivative of the neural crest, and thus they were referred to as lipomas of the IAC/CPA. However, research has since shown that these tumors arise from mesenchyme endogenous to the vestibulocochlear nerve and thus are more appropriately characterized as lipomatous choristomas. ${ }^{4}$ This theory offers an explanation for the failure of hearing conservation reported after surgical resection of these lesions. Patients with lipochoristomas may present with hearing loss, vestibular symptoms, or tinnitus. In this report we examine the appropriate work-up, diagnosis, and management of lipochoristoma of the IAC/CPA. received

February 9, 2014 accepted after revision

October 15, 2014

published online

March 4, 2015
DOI http://dx.doi.org/

10.1055/s-0034-1396654. ISSN 2193-6366. (c) 2015 Georg Thieme Verlag KG Stuttgart · New York
License terms

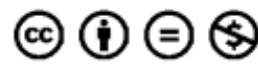


Table 1 Imaging characteristics of cerebellopontine angle lesions

\begin{tabular}{|l|l|l|l|}
\hline Mass & T1 & T1+ contrast & Notes \\
\hline Lipochoristoma & Hyperintense & No enhancement & T1 signal disappears with fat suppression \\
\hline Schwannoma & Isointense to brain & Enhances & \\
\hline Meningioma & Isointense to brain & Enhances & May have dural tail, calcification, or bony changes seen on CT $^{5}$ \\
\hline Epidermoid & Hypointense & No enhancement & Restricted diffusion on DWI $^{6}$ \\
\hline Metastatic disease & Variable & Enhances & No pathognomonic imaging characteristics \\
\hline
\end{tabular}

Abbreviations: CT, computed tomography; DWI, diffusion-weighted imaging.

\section{Results}

\section{Clinical Case}

A 51-year-old woman presented with 1 year of progressive left-sided severe hearing loss accompanied by tinnitus and weekly episodes of mild vertigo. In addition, the patient reported chronic headaches and intermittent ipsilateral facial paresthesias. She did not report facial weakness. Her initial audiogram demonstrated a left-sided severe sensorineural hearing loss with a word recognition score of $30 \%$; her right

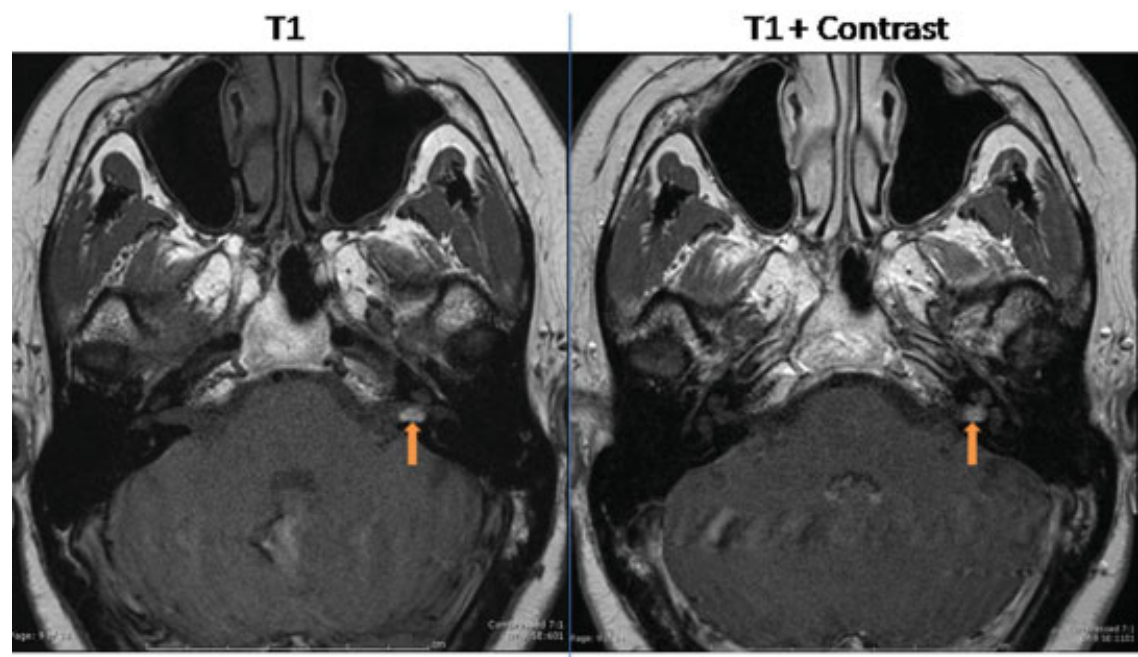

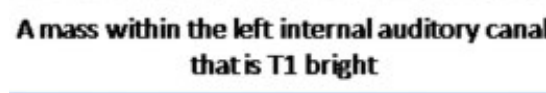

T1 with fat suppression

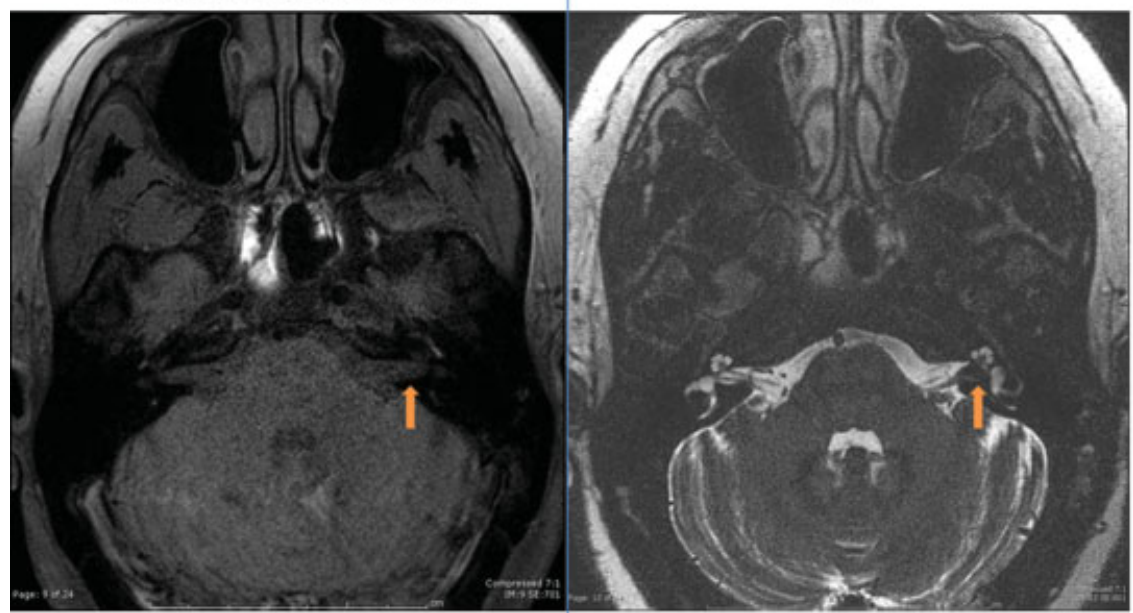

On fatsuppression imaging, the mass is dark
The mass does not enhance further with gadolinium contrast

T2

On high-definition T2 imaging (DRIVE), the mass is visible in the fundus of the IAC

Fig. 1 Lipochoristoma of the internal auditory canal (IAC). 
ear demonstrated normal hearing. An MRI obtained at an outside institution was read as a "small enhancing intracanalicular acoustic neuroma on the left." Initial review of this MRI demonstrated a small $(5 \times 4 \mathrm{~mm}) \mathrm{T} 1$ hyperintense lobulated lesion in the distal IAC. It was recommended that the patient undergo an interval MRI with gadolinium. With fat-suppressed images, there was no enhancement of the lesion, and the diagnosis of IAC lipochoristoma was confirmed. Conservative management was recommended, and on 17-month follow-up there has been no interval growth. The patient remains symptomatically stable with improved equilibrium but persistent left-sided hearing loss. She is currently considering a bone-anchored hearing aid.

\section{Discussion}

Tumors of the CPA and IAC may present with hearing loss, vestibular complaints, and facial paresthesias. Distinguishing between the types of lesion present in the IAC/CPA is possible with MRI imaging and additional specific sequences such as fat suppression. A comprehensive understanding of the differing imaging characteristics is of crucial importance to the otolaryngologist reviewing the imaging. - Table 1 depicts widely established imaging criteria for the most common IAC/CPA masses.

Lipochoristomas may mimic schwannomas, and care must be taken to look at various imaging modalities. ${ }^{7}$ Lipochoristomas normally display hyperintensity on T1-weighted images, which is atypical for schwannomas and raises clinical suspicion. However, lipochoristomas can occasionally have an isointense or hypointense appearance on T1. Therefore, T1-weighted images alone are not adequate to establish a diagnosis. In addition, care must be taken when interpreting a T1-weighted image postgadolinium. Enhancement in this setting, seen with schwannomas but not lipochoristomas, is defined by an increase in signal intensity compared with the T1-weighted image. Therefore, diagnosis is made with T1-weighted images that show fat suppression ${ }^{3}$ (-Fig. 1).

Lipochoristomas (lipomatous choristomas) classically were thought to arise from cells of the meninx primitiva, the mesenchymal derivative of the neural crest, which underwent abnormal differentiation. ${ }^{3}$ As such, they were classically and still are often erroneously referred to as "lipomas" of the IAC/CPA.

The clinical observation that these tumors are intimately associated with the auditory nerve was reported by Bigelow et al in $1998 .{ }^{8}$ This review of all 84 documented cases of lipomas of the IAC/CPA showed that although surgical resection had been performed in $62 \%$, total tumor resection was accomplished in only 17 of 52 . Furthermore, $68 \%$ of patients experienced postoperative complications, most commonly hearing loss (64\%). Facial paresis and other cranial neuropathies were also reported in this series. Despite Bigelow's report, a 2013 retrospective cohort study published in Laryngoscope continued to refer to these lesions as lipomas, despite the authors' comments that these tumors exhibit a "unique behavior" by "frequently engulfing coursing neurovascular structures."
In 2003, Wu et al presented a series of 11 cases that further elucidated the histology of these tumors. ${ }^{4}$ This offered an evidence-based explanation for the failure of hearing conservation by surgical resection and is one of the main arguments for conservative management of these lesions.

The natural history of lipochoristomas appears to be one of indolent growth. Wu et al reported no cases of growth in lipochoristomas treated conservatively (partial excision or biopsy) in a 7-year follow-up period. ${ }^{4}$ Bigelow et al reported one case of growth in a 16-year-old boy of the 20 lipochoristomas with documented radiographic follow-up. ${ }^{8}$ White et al reported one case of growth from $2.9 \mathrm{~mm}$ to $5.6 \mathrm{~mm} 10$ years after diagnosis in an 8-year-old girl. ${ }^{9}$ Because these lesions are composed primarily of mature adipocytes, some authors have theorized that they would be most prone to growth during periods of greatest fluctuation of body fat. ${ }^{9}$

\section{Conclusion}

Recent histologic research has provided convincing evidence that fatty tumors of the CPA are more appropriately named lipchoristomas than lipomas. Because lipochoristomas may have a tendency for more indolent growth and intimate involvement of the auditory nerve, conservative management with interval imaging is recommended as the treatment of choice. Neurotologists should be aware that hearing preservation surgery is likely not to be successful given the relationship between the lesion and the auditory nerve. Surgical treatment is reserved for growing and symptomatic lesions or those with disabling vestibular symptoms.

\section{References}

1 Martuza RL, Parker SW, Nadol JB Jr, Davis KR, Ojemann RG. Diagnosis of cerebellopontine angle tumors. Clin Neurosurg 1985;32:177-213

2 Brackmann DE, Bartels LJ. Rare tumors of the cerebellopontine angle. Otolaryngol Head Neck Surg (1979) 1980;88(5): 555-559

3 Bonneville F, Sarrazin JL, Marsot-Dupuch K, et al. Unusual lesions of the cerebellopontine angle: a segmental approach. Radiographics 2001;21(2):419-438

4 Wu SS, Lo WWM, Tschirhart DL, Slattery WH III, Carberry JN, Brackmann DE. Lipochoristomas (lipomatous tumors) of the acoustic nerve. Arch Pathol Lab Med 2003;127(11):1475-1479

5 Hamilton BE, Salzman KL, Patel N, et al. Imaging and clinical characteristics of temporal bone meningioma. AJNR Am J Neuroradiol 2006;27(10):2204-2209

6 Dutt SN, Mirza S, Chavda SV, Irving RM. Radiologic differentiation of intracranial epidermoids from arachnoid cysts. Otol Neurotol 2002;23(1):84-92

7 Wiggins RH III, Harnsberger HR, Salzman KL, Shelton C, Kertesz TR, Glastonbury CM. The many faces of facial nerve schwannoma. AJNR Am J Neuroradiol 2006;27(3):694-699

8 Bigelow DC, Eisen MD, Smith PG, et al. Lipomas of the internal auditory canal and cerebellopontine angle. Laryngoscope 1998; 108(10):1459-1469

9 White JR, Carlson ML, Van Gompel JJ, et al. Lipomas of the cerebellopontine angle and internal auditory canal: primum non nocere. Laryngoscope 2013;123(6):1531-1536 